\title{
An Automatic Spectrophotometric Titration Procedure for Ascorbic Acid Determination in Fruit Juices and Soft Drinks Based on Volumetric Fraction Variation
}

\author{
Ana Paula S. PAIM and Boaventura F. ReIS ${ }^{\dagger}$ \\ Centro de Energia Nuclear na Agricultura, Universidade de São Paulo, PO Box 96, \\ 13400-970 Piracicaba-SP, Brazil
}

\begin{abstract}
A spectrophotometric flow titration procedure for ascorbic acid in fruit juices and soft drinks based on volumetric fraction variation employing a reaction with 2,6-dichloroindophenol is described. A flow network was designed to implement the multicommutation process and a set of microcomputer controlled three-way solenoid valves was employed to handle the insertion of the titrand and titrant solutions into the analytical path. All the steps involved in the titration procedure were carried out automatically without any operator assistance. Fruit juices and soft drinks could be analyzed without any prior chemical treatment. Accuracy was assessed by comparing results with those obtained by the reference method (AOAC). No significant differences at $95 \%$ confidence level were observed. Other profitable features were achieved such as a percentual standard deviation of results of $1.1 \%(n=5)$ for a typical juice sample containing $1.2 \mathrm{mmol} \mathrm{L}^{-1}$ ascorbic acid, a low sample consumption ( $6 \mu \mathrm{L}$ per run), and an analytical throughput ranging from 5 to 30 determinations per hour for sample concentrations ranging from 0.6 to $6.0 \mathrm{mmol} \mathrm{L}^{-1}$.
\end{abstract}

(Received July 22, 1999; Accepted February 9, 2000)

\begin{abstract}
Ascorbic acid is widely utilized as an additive in juices, sweet drinks and foods, and is also used in pharmaceutical preparations such as vitamins. Sometimes, its addition is carried out during product manufacturing in order to increase the nutritional value or as an anti-oxidant to lengthen the lifetime of the industrialized products. In this sense, ascorbic acid is present in several interesting matrices including biologic fluids such as blood and urine. ${ }^{1}$ Concerning the matrix diversity, several analytical procedures have been proposed for ascorbic acid determination, and those based on flow analysis should be mentioned. ${ }^{1-11}$ Facilities to implement the analytical procedure, high throughput and low reagent consumption, could be considered as advantages provided by the flow analysis technique. In this sense, spectrophotometry, ${ }^{2-5}$ chemiluminescence, ${ }^{1,6}$ amperometry ${ }^{7,8}$ and potentiometry ${ }^{11}$ have been employed as detection techniques.

Sample analysis without any prior chemical treatment should be desirable in order to decrease sample handling steps while also saving time of analysis. These facilities can be provided by exploiting the multicommutation and binary sampling process considering its ability to handle low volumes of solution. ${ }^{12,13}$ Nevertheless, the coloration in some natural juices could hamper the spectrophotometic determination. ${ }^{14}$

Titration is an old analytical process which is widely employed today in routine analysis as well as in the development of new analytical methodologies. Several procedures based on the flow injection technique have been proposed employing spectrophotometry ${ }^{13,15-17}$ or potentiometry ${ }^{18-20}$ as detection techniques. Most of the flow procedures employed mixing chamber $^{16-18}$ or variation in reagent flow rate ${ }^{15,19}$ as strategies to generate gradients of concentration. In both cases, analytical
\end{abstract}

† To whom correspondence should be addressed.

E-mail: reis@cena.usp.br curves were required to determine the analyte concentration and to do this a set of standard solutions is run prior to the sample processing.

In this work, we intend to develop an automatic titrimetric flow procedure exploiting multicommutation and binary sampling process to implement the volumetric fraction variation of the titrant solution in order to find the end point of titration. The procedure will be applied for the determination of ascorbic acid in fruit juices (in nature) and soft drinks. Spectrophotometry is employed as the detection technique. The method is based on the reaction of ascorbic acid with 2,6dichloroindophenol. ${ }^{21}$

A desirable feature that should be provided by an automatic titration procedure is the ability to work by using a titrant solution volume lower or higher than that of the sample solution. With this feature, samples presenting a wide concentration range could be analyzed using a single titrant solution. A flow system based on multicommutation can provide the facility to handle small solution volumes. ${ }^{12}$ This feature will be exploited in this work to widen the sample concentration range that could be titrated by using a single titrant solution.

\section{Experimental}

\section{Solutions}

All chemicals were of analytical reagent grade. Distilled and deionized water was used throughout.

A $0.43 \mathrm{mmol} \mathrm{L} \mathrm{L}^{-1}$ 2,6-dichloroindophenol was prepared by dissolving $25 \mathrm{mg}$ of its sodium salt (Sigma EEC No 210-640-4). This was done by placing the mass of salt into a beaker, adding $50 \mathrm{~mL}$ of water plus $21 \mathrm{mg} \mathrm{NaHCO}_{3}$ to it, and stirring with a magnetic bar up to perfect dissolution; then the volume was 

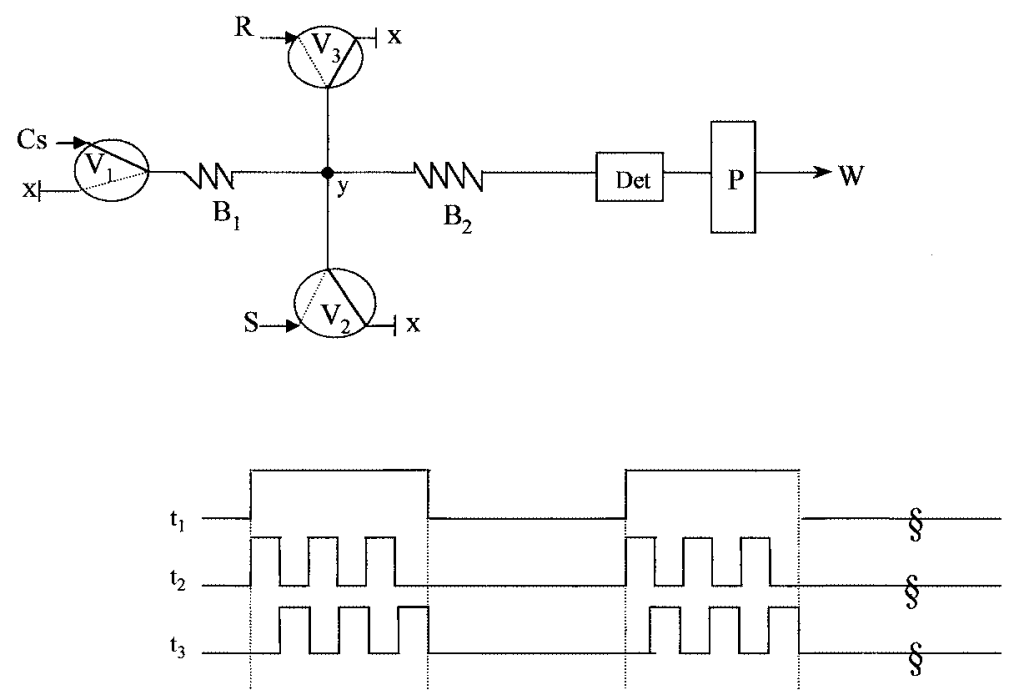

Fig. 1 Flow diagram of the system. $V_{1}, V_{2}$ and $V_{3}=$ three-way solenoid valves; $B_{1}$ and $B_{2}=10$ and $25 \mathrm{~cm}$ straight PTFE tubing and $1.6 \mathrm{~mm}$ i.d.; Det $=$ spectrophotometer at $526 \mathrm{~nm} ; \mathrm{P}=$ peristaltic pump, flow rate at $1.8 \mathrm{~mL} \mathrm{~min}^{-1} ; \mathrm{S}=$ sample; $\mathrm{Cs}=$ water; $\mathrm{R}=2,6$-dichloroindophenol; $\mathrm{x}=$ valve inlet stopper; $\mathrm{y}=$ confluence point; $\mathrm{W}=$ waste. Solid line inside valve symbol indicates flow pathway for valve off, and dashed line indicates flow pathway for valve on; $t_{1}, t_{2}, t_{3}=$ valve switching timing course; high level indicates valve switched on.

made up to $200 \mathrm{~mL}$ with water. This solution was stored in amber bottle to protect against light and stored in a refrigerator when not in use. This solution could be used for a week.

A $0.375 \mathrm{~mol} \mathrm{~L}^{-1}$ metaphosphoric acid solution was prepared by dissolving $15 \mathrm{~g} \mathrm{HPO}_{3}$ pellets in $40 \mathrm{~mL}$ glacial $\mathrm{CH}_{3} \mathrm{COOH}$ plus $200 \mathrm{~mL}$ of water. After dissolution, the volume was made up to $500 \mathrm{~mL}$ with water. This solution was stored in a refrigerator when not being used.

A $6.0 \mathrm{mmol} \mathrm{L}^{-1}$ ascorbic acid standard solution was prepared daily by dissolving $50 \mathrm{mg}$ ascorbic acid (Merck) in $50 \mathrm{~mL}$ of the metaphosphoric acid plus acetic acid solution as described in the last paragraph.

Sample preparation was accomplished by adding $15.0 \mathrm{~mL}$ of the $\mathrm{HPO}_{3}-\mathrm{CH}_{3} \mathrm{COOH}$ solution to $10.0 \mathrm{~mL}$ of the sample. When kept in a refrigerator, this solution could be used for a week.

The orange and lime juices were prepared by squeezing the fruit; afterwards each juice was filtered (Whatman, Ashless 44). A $10 \mathrm{~mL}$ aliquot was transferred to a volumetric flask and the volume was made up to $25 \mathrm{~mL}$ with the $\mathrm{HPO}_{3}-\mathrm{CH}_{3} \mathrm{COOH}$ solution.

\section{Apparatus}

The equipment consisted of a 435 Femto spectrophotometer with a flow cell of $100 \mu \mathrm{L}$ (12 mm optical path) at $526 \mathrm{~nm}$, an IPC-4 Ismatec peristaltic pump with Tygon pumping tube, a 486 microcomputer equipped with a PCL-711S Advantech electronic interface card and running software written in Quick Basic 4.5. The flow network comprised a set of three-way solenoid valves (161T031, NResearch), reaction coils of PTFE tubing (i.d. $0.7 \mathrm{~mm}$ ) and four-way joints machined in Perspex. To match the electric current intensity and the potential difference between the PCL-711S interface card and the threeway solenoid valves, a home-made electronic interface was employed. ${ }^{12}$ To allow the control of the peristaltic pump, it was linked to the microcomputer through the RS232 serial interface. To minimize pulsation effects due to pumping flow pattern, the tachometer signal generated by the peristaltic pump was used as a reference to synchronize the pulsation pattern with sampling cycle. For this, the tachometer output was coupled to the analog input $\left(\mathrm{A} / \mathrm{D}_{1}\right)$ of the PCL-711S interface card.

\section{Procedure}

A flow system was designed to implement the multicommutation and binary sampling approach. Its flow diagram is depicted in Fig. 1. In this configuration, all valves are switched off and only the carrier solution (Cs) is flowing through the reaction coil $\left(\mathrm{B}_{2}\right)$. The software control all steps of the titration procedure and its flow chart is shown in Fig. 2. When the software was initialized, the microcomputer requested the control data which are summarized in Table 1. Prior to beginning the titration procedure, the microcomputer sent the starting command to the peristaltic pump and waited for the synchronization signal coming from it. Thereafter, the microcomputer sends an electric pulse to $\mathrm{V}_{1}$ and $\mathrm{V}_{2}$ valves to switch them on during a time interval $\Delta t_{\mathrm{s}}$ (Fig. 1). While these valves are kept on, the carrier stream (Cs) was halted and a slug of the titrand solution (S) with a volume $v_{\mathrm{s}}=\varphi \cdot \Delta t_{\mathrm{s}}(\varphi=$ flow rate) was inserted into the reaction coil $B_{2}$. Afterwards, $V_{2}$ valve was switched off and $V_{1}$ and $V_{3}$ valves were switched on during a time interval $\Delta t_{\mathrm{t}}$ to insert into the reaction coil $\mathrm{B}_{2}$ a slug of the titrant solution with a volume $v_{\mathrm{t}}=\varphi \cdot \Delta t_{\mathrm{t}}$. These two steps, referred as a sampling cycle can be repeated several times $\left(n_{\mathrm{s}}\right)$ to load coil $B_{2}$ with a string comprising slugs of the titrand solution in tandem with slugs of the titrant solution. When sampling cycle was ended, all valves were switched off and carrier solution flowed again to transport the sample zone through the coil $\mathrm{B}_{2}$ towards the detector (Det). The number of the sampling cycle $\left(n_{\mathrm{s}}\right)$ can be changed in order to control the dispersion of the sample zone in the carrier stream. The ratio between slug volumes of sample and titrant solutions can be varied to attain the end point of the titration.

Prior to starting the titration procedure, $\mathrm{V}_{1}$ and $\mathrm{V}_{2}$ valves were switched on during a time interval $t=n_{\mathrm{s}} \cdot \Delta t_{\mathrm{s}}$ (see Table 1) to insert sample volume $v=n_{\mathrm{s}} \cdot \varphi \cdot \Delta t_{\mathrm{s}}$ into the coil $\mathrm{B}_{2}$. The signal generated by the detector in this run was read by the microcomputer; its average and standard deviation, which are 


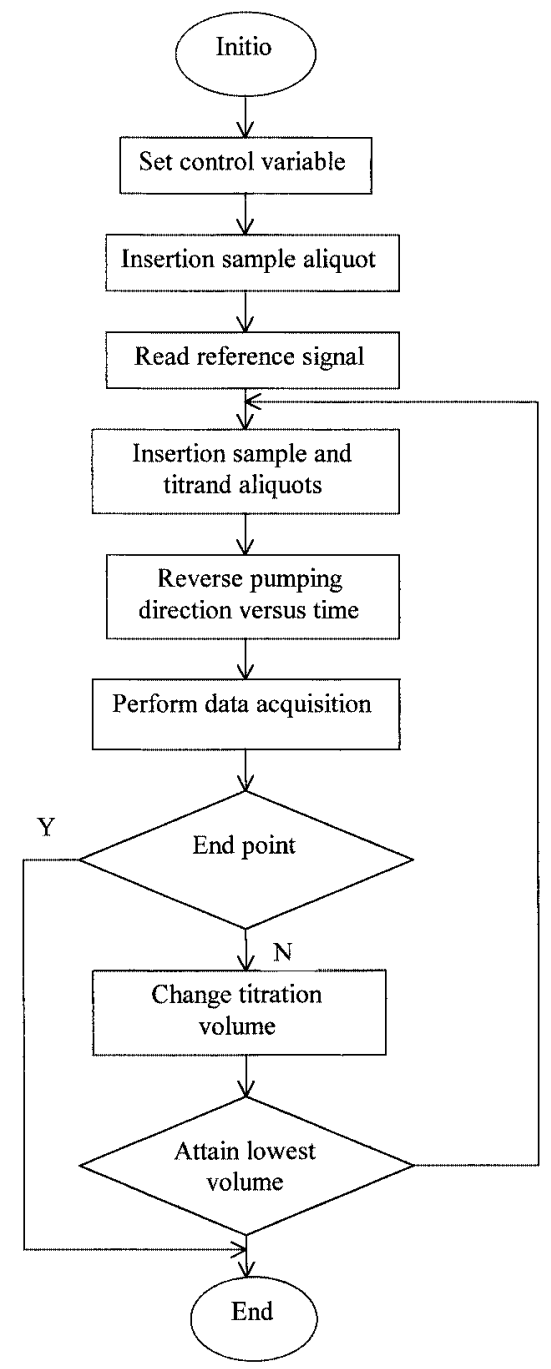

Fig. 2 Flow chart of the control software.

here abbreviated as $S_{0}$ and $d_{0}$, were estimated and employed as references to determine the end point of the titration.

In the first titration run, $n_{\mathrm{s}}$ sampling cycles comprising the time intervals $\Delta t_{\mathrm{s}}$ and $\Delta t_{\mathrm{t}}$ were carried out in order to insert slugs of sample and of titrant solutions, respectively $\left(\Delta t_{\mathrm{t}}=\Delta t_{\mathrm{s}} / 2\right)$. A portion of the reaction coil $\mathrm{B}_{2}$ was loaded with a string comprising slugs of sample solution in tandem with slugs of titrant solution. After the loading steps, all valves were switched off and the pumping was reversed during a $\Delta t_{\mathrm{f}}$ time interval. Afterwards, the original pumping condition was established again and after a time interval $t=\Delta t_{\mathrm{r}}$ the pumping forward was stopped to begin another reversing cycle.

The reverse cycle was implemented to improve mixing between sample and titrant solutions and was repeated $n_{\mathrm{r}}$ times. This task was done by the microcomputer sending a control command to the peristaltic pump through the serial interface. After the pump reversal cycles $\left(n_{\mathrm{r}}\right)$ were performed, the forward pumping was restored to direct product of reaction towards the detector (Det).

The analytical signal was read as a time function by the microcomputer through the analog input of the PCL-711S interface card. After reading the analytical signal, the microcomputer increased the pumping flow rate to perform the washing step (see Table 1).

The maximum value of the analytical signal $\left(S_{\max }\right)$ was
Table 1 Software control parameters

\begin{tabular}{|c|c|c|c|c|c|}
\hline Step & $\begin{array}{c}\text { Flow rate/ } \\
\mu \mathrm{L} \mathrm{s}^{-1}\end{array}$ & $\begin{array}{l}\text { Valve } \\
\text { on }\end{array}$ & $\begin{array}{c}\text { Time/ } \\
\mathrm{s}\end{array}$ & $\begin{array}{l}\text { Sampling } \\
\text { cycle }\end{array}$ & $\begin{array}{l}\text { Signal } \\
\text { limit }\end{array}$ \\
\hline Load sample & 30 & $V_{1}+V_{2}$ & $\Delta t_{\mathrm{s}}(2.0)$ & $n_{\mathrm{s}}(4)$ & - \\
\hline $\begin{array}{l}\text { Load titrant } \\
\text { First run }\end{array}$ & 30 & $V_{1}+V_{3}$ & $\Delta t_{\mathrm{t}}(1.0)$ & - & - \\
\hline Reverse flow & 30 & - & $\Delta t_{\mathrm{f}}(3.0)$ & $n_{\mathrm{r}}(3)$ & - \\
\hline Read signal & 30 & - & $\Delta t_{\mathrm{r}}(15.0)$ & & - \\
\hline Wash & 60 & - & $\Delta t_{\mathrm{w}}(10.0)$ & - & - \\
\hline Increment & - & - & $\Delta t_{\mathrm{i}}(0.2)$ & - & $L_{\min }(5)$ \\
\hline Decrement & & & $\Delta t_{\mathrm{d}}(0.2)$ & & $L_{\max }(50)$ \\
\hline Lowest volume & - & - & $\Delta t_{\mathrm{v}}(0.1)$ & - & - \\
\hline $\begin{array}{l}\text { Lowest } \\
\text { variation }\end{array}$ & - & - & $\Delta t_{\mathrm{c}}(0.05)$ & - & - \\
\hline
\end{tabular}

Numbers inside parentheses are the selected values.

selected and compared with the reference signal $\left(S_{0}\right)$ which was obtained prior to beginning the titration using the following equations:

$$
\begin{aligned}
& S_{\max }>S_{0}+d_{0} \cdot L_{\max } \\
& S_{\max }<S_{0}+d_{0} \cdot L_{\text {min }}
\end{aligned}
$$

The condition expressed by Eq. (1) indicated a titrant excess in the sample zone and that expressed by Eq. (2) indicated a titrand excess. In the first situation, the volume of the titrant solution slug was decreased prior to carrying out the next run, while in the second one it was increased. In this sense, the time interval to switch $\mathrm{V}_{1}$ and $\mathrm{V}_{3}$ valves on was settled to $\Delta t_{\mathrm{t}}=\Delta t_{\mathrm{x}}+$ $\Delta t_{\mathrm{i}}$ or $\Delta t_{\mathrm{t}}=\Delta t_{\mathrm{x}}-\Delta t_{\mathrm{d}}$, where $\Delta t_{\mathrm{x}}$ was the last time interval employed to switch on these valves (see Table 1). The maximum $\left(L_{\max }\right)$ and minimum $\left(L_{\min }\right)$ limits are arbitrary parameters and were established as 5 and 50, respectively.

The titration procedure continues up to attain the end point maintaining the time interval $\Delta t_{\mathrm{s}}$ to switch on $\mathrm{V}_{1}$ and $\mathrm{V}_{2}$ valves and varying $\Delta t_{\mathrm{t}}$ to switch on $\mathrm{V}_{1}$ and $\mathrm{V}_{3}$ valves as described in the last paragraph. In this sense, the sample aliquot was constant while the titrant solution volume was varied, since for each run the time interval $\Delta t_{\mathrm{t}}$ presented a different value. After each analytical run, the collected data were processed to decide about the titration course to be followed in the next analytical run; also, these data were saved in a file to allow further treatment. Titration ended when the analytical signal $\left(S_{\max }\right)$ was lower than $S_{0}+d_{0} \cdot L_{\max }$ and higher than $S_{0}+d_{0} \cdot L_{\min }$, or when the time interval $\Delta t_{\mathrm{t}}$ to switch $\mathrm{V}_{1}$ and $\mathrm{V}_{3}$ valves on was equal to $\Delta t_{\mathrm{s}}$ or equal to $\Delta t_{\mathrm{v}}$.

The system parameters such as sampling flow rate, slug volume of sample solution, number of sampling cycles, number and duration of reversing cycles, and analyte concentration range were investigated. After these parameters have been established, samples of fruit juices and soft drinks were analyzed.

\section{Results and Discussion}

In the presence of ascorbic acid, the color of titrant (2,6dichloroindophenol) vanished due to reduction caused by this chemical species. ${ }^{22}$ This feature was exploited to detect the end point titration. As indicated in Fig. 3, when an excess of the titrant remained in the sample bulk, a signal higher than the baseline was detected.

In Fig. 3, the reference signal $\left(S_{0}\right)$ and its standard deviation 


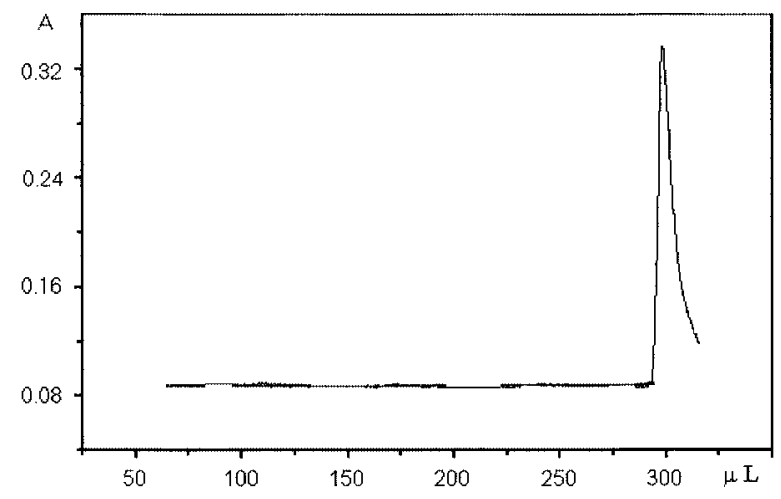

Fig. 3 Record of the titration. $A=$ absorbance. Figure refers to the system of Fig. 1 with: flow rate $=30 \mu \mathrm{L} \mathrm{s}^{-1}$; sample loading time, $\Delta t_{\mathrm{s}}$ $=1 \mathrm{~s}$; sampling cycle, $n_{\mathrm{s}}=8$. A $1.2 \mathrm{mmol} \mathrm{L}^{-1}$ ascorbic acid solution and a $0.43 \mathrm{mmol} \mathrm{L}^{-1}$ titrant solution.

$\left(d_{0}\right)$ were in absorbance units 0.088 and 0.002 , respectively. Therefore, considering the values of the parameters $\max$ and min settled in Table 1, the titration should be ended if the analytical signal $S_{\max }$ was higher than 0.097 or lower than 0.176 . To define the value of the parameters $\max$ and $\min$, several experiments were performed by using ascorbic acid concentrations of 0.6 and $6.0 \mathrm{mmol} \mathrm{L}^{-1}$. To minimize noise effect which could occur, prior to selecting the maximum signal $\left(S_{\max }\right)$, the data were smoothed by applying the moving average with five levels.

In the proposed flow system, sample and titrant solutions were inserted into the reaction coil as a string of sample slugs in tandem with slugs of titrant solution, as depicted in Fig. 1. Under this condition, mixing between solutions occurred by dispersion at part from the mutual liquid interfaces; therefore the dimensions and geometry of the reactor could exert a remarkable effect on the mixing efficiency. Two types of reactors were tested: a coiled tube, $100 \mathrm{~cm}$ long and $0.8 \mathrm{~mm}$ i.d. $(500 \mu \mathrm{L})$; and a straight tube, $25 \mathrm{~cm}$ long and $1.6 \mathrm{~mm}$ i.d. $(500 \mu \mathrm{L})$.

The relative standard deviation of the results was selected as a parameter to select the reactor to be employed. The precision of results obtained with the coiled reactor was less (relative standard deviation >0.02) when the sample slug volume was larger than $10 \mu \mathrm{L}$, thus indicating a poor mixing condition. Since the lowest volume of the titrant solution slug was settled at $3.0 \mu \mathrm{L}\left(\Delta t_{\mathrm{t}}=0.1 \mathrm{~s}\right)$, thus using sample slugs with volumes of $10 \mu \mathrm{L}$ could make the titration range become very narrow. Employing the straight tubing as reactor and reversing the pumping direction 3 times during $2 \mathrm{~s}$, we obtained results with relative standard deviations lower than 0.014 . In this case, the sample slug volume was settled at $60 \mu \mathrm{L}\left(\Delta t_{\mathrm{s}}=2.0 \mathrm{~s}\right)$ and four sampling cycles $\left(n_{\mathrm{s}}=4\right)$ were carried out; thus the sample solution volume used for each analytical run was $240 \mu \mathrm{L}$. The volume of the titrant solution inserted to carry out the first run was $120 \mu \mathrm{L}\left(\Delta t_{\mathrm{t}}=\Delta t_{\mathrm{s}} / 2\right)$. According to $S_{\max }$ value, the slug volume of the titrant solution was increased or decreased in step of $6.0 \mu \mathrm{L}$ up to attain the end point condition $\left(S_{0}+d_{0}\right.$. $\left.L_{\min }\right)<S_{\max }<\left(S_{0}+d_{0} \cdot L_{\max }\right)$ as previously defined. Afterwards, an additional run was carried out to perform a fine adjusting. This was done by settling the titrant loading time interval to $\Delta t_{\mathrm{t}}=\Delta t_{\mathrm{x}}$ $-0.1 \mathrm{~s}$. If $\left(S_{0}+d_{0} \cdot L_{\min }\right)<S_{\max }$, then, this loading time was utilized to calculate the titrant solution volume; otherwise, if this condition was not achieved, the penultimate time interval was considered. In this sense, the smallest slug volume variation was $3.0 \mu \mathrm{L}$ and was employed to improve accuracy.

After the system parameters were established (Table 1), a set
Table 2 Results comparison: data, in $\mathrm{mg} \mathrm{L}^{-1}$ ascorbic acid, are results obtained with the proposed and reference methods

\begin{tabular}{lcc}
\hline Sample & $\begin{array}{c}\text { Proposed procedure/ } \\
\mathrm{mg} \mathrm{L}^{-1}\end{array}$ & $\begin{array}{c}\text { Reference method/ } \\
\mathrm{mg} \mathrm{L}^{-1}\end{array}$ \\
\hline Cashew fruit juice $^{\mathrm{a}}$ & $390 \pm 0$ & $391 \pm 2$ \\
Guava juice $^{\mathrm{a}}$ & $380 \pm 0$ & $380 \pm 0$ \\
Orange juice $^{\mathrm{b}}$ & $215 \pm 1$ & $218 \pm 2$ \\
Lemon juice $^{\mathrm{b}}$ & $95 \pm 0$ & $89 \pm 4$ \\
Soft drink 1 $_{\text {Soft drink 2 }}$ & $45 \pm 1$ & $45 \pm 2$ \\
Isotonic drink & $37 \pm 2$ & $42 \pm 2$ \\
\hline
\end{tabular}

Estimation of standard deviation for $n=3$. a. Industrialized juices.

b. Natural juices.

of fruit juices and soft drinks were analyzed, yielding the results shown in Table 2. Accuracy was assessed by applying the paired $t$-test to the results and those obtained with the reference method ${ }^{21}$ no significant difference at $95 \%$ confidence level was observed.

Flow rate and sample loading time $\left(\Delta t_{\mathrm{s}}\right)$ were maintained at 30 $\mu \mathrm{L} \mathrm{s}^{-1}$ and $2 \mathrm{~s}$, respectively, then a sample volume of $240 \mu \mathrm{L}$ was used to carry out each analytical run. In this sense, if the titration end point was detected in the first run, the volumes of the sample and titrant solutions used were 240 and $120 \mu \mathrm{L}$, respectively. Nevertheless, if titration was ended with the lowest volume of the titration solution $\left(\Delta t_{\mathrm{t}}\right.$ changing from 1.0 up to 0.1 s), the volumes of titrand and titrant solutions were 372 and $1440 \mu \mathrm{L}$, respectively. On the other hand, if titration required the largest volume of the titrant solution $(240 \mu \mathrm{L})$ the overall volumes of the solutions used were 1080 and $1440 \mu \mathrm{L}$ for titrant and titrand solutions, respectively. This volume of sample solution was $28 \%$ lower than that used to carried out the reference method,22 thus decreasing also the volume of the titrand solution. Under this condition, and using a $0.43 \mathrm{mmol} \mathrm{L}^{-1}$ titrant solution concentration, samples with ascorbic acid concentrations within 0.6 and $6.0 \mathrm{mmol} \mathrm{L}^{-1}$ could be titrated. Depending on the sample concentration, the number of titration runs could be varied from 3 to 7 ; therefore the time interval needed to perform the titration could be varied from 129 to $281 \mathrm{~s}$.

Sample solutions were processed without any previous treatment and analyte concentrations were determined without using analytical curves. The system's long-term stability was verified by running it continuously during $4 \mathrm{~h}$ and no drift was observed. This test was repeated several days using standard solution; results presented a relative standard deviation lower than $2 \%$, indicating a good robustness. The system operation is very simple, which can be considered an additional advantage.

\section{Acknowledgement}

The authors are grateful to CAPES, FAPESP, CNPq and FINEP/PRONEX for financial support and for a grant to Ana Paula S. Paim, and also to Dr. E. A. G. Zagatto for commenting on the manuscript.

\section{References}

1. A. A. Alwarthan, Analyst, 1993, 118, 639.

2. S. M. Sultan, Talanta, 1993, 40, 593.

3. J. A. Nóbrega and G. S. Lopes, Talanta, 1996, 43, 971.

4. S. M. Sultan and N. I. Desai, Talanta, 1998, 45, 1061. 
5. J. M. Alamo, A. Maquieira, R. Puchades, and S. Sagrado, Fresenius J. Anal. Chem., 1993, 347, 293.

6. J. M. Kim, Y. Huang, and R. D. Schmidt, Anal. Lett., 1990 , 23, 2273.

7. A. G. Fogg, A. M. Summan, and M. A. FernandezArciniega, Analyst, 1985, 110, 341.

8. A. M. Almuaibed and A. Townshend, Talanta, 1992, 11, 1459.

9. L. E. Léon and J. Catapano, Anal. Lett., 1993, 26, 1741.

10. A. S. Martínez, A. Ríos, and M. Valcárcel, Analyst, 1992, 117, 1761.

11. J. Li, G. Shen, and R. Yu, Analyst, 1995, 120, 2259.

12. B. F. Reis, M. F. Giné, E. A. G. Zagatto, J. L. F. C. Lima, and R. A. Lapa, Anal. Chim. Acta, 1994, 293, 129.

13. M. Korn, L. F. B. P. Gouveia, E. Oliveira, and B. F. Reis, Anal. Chim. Acta, 1995, 313, 177.
14. A. Safavi and L. Fotouhi, Talanta, 1994, 41, 1225.

15. J. Marcos, A. Ríos, and M. Valcárcel, Anal. Chim. Acta, 1992, 261, 495.

16. F. Sagara, T. Kobayashi, T. Tajima, H. Ijyuin, I. Yoshida, D. Ishii, and K. Ueno, Anal. Chim. Acta, 1992, 261, 505.

17. A. Tan and C. Xiao, Anal. Chim. Acta, 1997, 341, 297.

18. S. F. Simpson and F. J. Holler, Anal. Chem., 1982, 54, 43.

19. J. Bartrolí, L. Alerm, J. García-Raurich, and J. Masip, Quim. Anal., 1994, 13, 31.

20. P. B. Martelli, B. F. Reis, M. Korn, and J. L. F. C. Lima, Anal. Chim. Acta, 1999, 387, 165.

21. Official Methods of Analysis of Association Official Analytical Chemists, ed. S. Williams, 14th ed., 1995, sec. 45. 1. 14, chap. 45, Arlington, 16.

22. W. L. Hall and M. J. Deutsch, J. Assoc. Off. Anal. Chem., $\mathbf{1 9 6 5}, 48,1236$. 